Turk. J. Math. Comput. Sci.

13(2)(2021) 63-67

(C) MatDer

DOI : $10.47000 /$ tjmcs. 835237

\title{
De Moivre-Type Identities for the Pell Numbers
}

\author{
Seda Yamaç Akbiyik ${ }^{1, *}$ (D), Mücahit Akbiyik ${ }^{2}$ (D) \\ ${ }_{1, *}$ Department of Computer Engineering, Faculty of Engineering and Architecture, Istanbul Gelisim University, 34310, Avcilar, \\ Istanbul, Turkey. \\ ${ }^{2}$ Department of Mathematics, Faculty of Arts-Sciences, Beykent University, 34500, Büyükçekmece, Istanbul, Turkey.
}

Received: 03-12-2020 • Accepted: 23-03-2021

Aвstract. This paper aims to present a method for constructing the second order Pell and Pell-Lucas numbers and the third order Pell and Pell-Lucas numbers. Moreover, we obtain the De Moivre-type identities for these numbers. In addition, we define a Pell sequence with new initial conditions and give some identities for these third order Pell numbers such as Binet's formulas, generating functions, sums.

2010 AMS Classification: Primary 11B39, 05A15

Keywords: Pell numbers, Pell-Lucas numbers, De Moivre-type identity, binet's formula.

\section{INTRODUCTION}

In the literature, the roots of the equation $x^{2}-x-1=0$ are given as $x_{1}=(1+\sqrt{5}) / 2, x_{2}=(1-\sqrt{5}) / 2$, and the following relation is satisfied

$$
\left(\frac{1 \pm \sqrt{5}}{2}\right)^{n}=\frac{L_{n} \pm \sqrt{5} F_{n}}{2}
$$

where $L_{n}$ denotes the $n^{\text {th }}$ Lucas numbers and $F_{n}$ denotes the $n^{\text {th }}$ Fibonacci numbers for the integers $n$. The relation (1.1) is called De Moivre-type identity, [2]. In [9] and [10], Lin gave the De Moivre-type identities for Tribonacci and Tetrabonacci numbers by using the recurrence relation $x^{3}-x^{2}-x-1$ and the recurrence relation $x^{4}-x^{3}-x^{2}-x-1$, respectively.

In this paper, we give a way for constructing the second order Pell and Pell-Lucas numbers and the third order Pell and Pell-Lucas numbers by using the roots of characteristic equations $x^{2}-2 x-1=0$ and $x^{3}-2 x^{2}-x-1=0$, respectively. Moreover, we obtain the De Moivre-type identity for the second order Pell numbers and the third order Pell numbers. Furthermore, we define a third order Pell sequence with new initial conditions and find some identities between all of these sequences.

*Corresponding Author

Email addresses: syamac@gelisim.edu.tr (S. Yamaç Akbıyık), mucahitakbiyik@beykent.edu.tr (M. Akbıyık) 


\section{De Moivre-Type Identities for Pell numbers}

Horadam in [5], defines the Pell sequence with the recurrence relation

$$
P_{n+2}=2 P_{n+1}+P_{n}
$$

and with the initial conditions $P_{0}=0, P_{1}=1$, where $P_{n}$ denotes the $n^{\text {th }}$ Pell number. Some terms of $\left\{P_{n}\right\}$ are as follows

$$
\left\{P_{n}\right\}=0,1,2,5,12,29, \ldots
$$

Also, some identities for the Pell numbers are given by Horadam. In the literature, there are a lot of studies about Pell number such as [4,6-8] and [1]. The characteristic equation of the Pell sequence is $x^{2}-2 x-1=0$ and the roots of it are $r_{1}=1+\sqrt{2}, r_{2}=1-\sqrt{2}$, [5]. By calculating the powers of the roots, the De Moivre-type identity for the Pell numbers can be found as follows;

$$
(1 \pm \sqrt{2})^{n}=\frac{A_{n} \pm \sqrt{2} P_{n}}{2}
$$

analog to the De Moivre-type identity for Fibonacci numbers. Note that the sequence $\left\{A_{n}\right\}$ takes place in The On-Line Encyclopedia of Integer Sequences (OEIS, A002203) called as the Companion Pell numbers or Pell- Lucas numbers in [11] with the initial conditions $A_{0}=2, A_{1}=2$.

TABLE 1. Some terms of the sequences $\left\{A_{n}\right\}$ and $\left\{P_{n}\right\}$

\begin{tabular}{||cccccccccccc||}
\hline $\mathrm{n}$ & 0 & 1 & 2 & 3 & 4 & 5 & 6 & 7 & 8 & 9 & 10 \\
\hline \hline$A_{n}$ & 2 & 2 & 6 & 14 & 34 & 82 & 198 & 1154 & 2786 & 6726 & 16238 \\
\hline$P_{n}$ & 0 & 1 & 2 & 5 & 12 & 29 & 70 & 169 & 408 & 985 & 2378 \\
\hline
\end{tabular}

\section{De Moivre-Type Identities for the Third Order Pell Numbers}

In [12], the third order Pell numbers is defined and some identities for this numbers are found. We know from [12] that the recurrence relation for the third order Pell numbers is

$$
x^{3}-2 x^{2}-x-1=0 .
$$

Addition to this, the three roots of the equation (3.1) are

$$
\begin{gathered}
r_{1}=\frac{2}{3}+\left(\frac{61}{54}+\sqrt{\frac{29}{36}}\right)^{1 / 3}+\left(\frac{61}{54}-\sqrt{\frac{29}{36}}\right)^{1 / 3}, \\
r_{2}=\frac{2}{3}+\omega\left(\frac{61}{54}+\sqrt{\frac{29}{36}}\right)^{1 / 3}+\omega^{2}\left(\frac{61}{54}-\sqrt{\frac{29}{36}}\right)^{1 / 3}, \\
r_{3}=\frac{2}{3}+\omega^{2}\left(\frac{61}{54}+\omega \sqrt{\frac{29}{36}}\right)^{1 / 3}+\omega\left(\frac{61}{54}-\sqrt{\frac{29}{36}}\right)^{1 / 3},
\end{gathered}
$$

where $\omega=\frac{-1+i \sqrt{3}}{2},[12]$.

Let us rewrite the roots of equation (3.1) as follows:

$$
\begin{gathered}
r_{1}=\frac{2}{3}+X+Y, \\
r_{2}=\frac{2}{3}-\frac{1}{2}(X+Y)+\frac{\sqrt{3}}{2} i(X-Y), \\
r_{2}=\frac{2}{3}-\frac{1}{2}(X+Y)-\frac{\sqrt{3}}{2} i(X-Y),
\end{gathered}
$$

where $X=\sqrt[3]{\frac{61}{54}+\sqrt{\frac{29}{36}}}$ and $Y=\sqrt[3]{\frac{61}{54}-\sqrt{\frac{29}{36}}}$. Also, we know that $X . Y=\frac{7}{9}$ and $X^{3}+Y^{3}=\frac{61}{27}$ are satisfied. Thus,

$$
r_{1}^{2}=2+\frac{4}{3}(X+Y)+\left(X^{2}+Y^{2}\right),
$$




$$
\begin{aligned}
& r_{1}^{3}=\frac{17}{3}+\frac{11}{3}(X+Y)+2\left(X^{2}+Y^{2}\right), \\
& r_{1}^{4}=14+\frac{29}{3}(X+Y)+5\left(X^{2}+Y^{2}\right), \\
& r_{1}^{5}=\frac{107}{3}+\frac{73}{3}(X+Y)+13\left(X^{2}+Y^{2}\right), \\
& r_{1}^{6}=91+62(X+Y)+33\left(X^{2}+Y^{2}\right),
\end{aligned}
$$

The coefficients of the above equations construct the third order Pell sequences, which we denote by $\left\{R_{n}\right\},\left\{S_{n}\right\}$ and $\left\{T_{n}\right\}$, respectively. Especially,

(1) the sequence $\left\{R_{n}\right\}$ is the third order Pell-Lucas in [12],

(2) the sequence $\left\{S_{n}\right\}$ is the third order Pell numbers sequence with the initial conditions $S_{0}=3, S_{1}=4$ and $S_{3}=11$,

(3) the sequence $\left\{T_{n}\right\}$ is the usual third order Pell sequence which is also called as Tripell sequence or the sequence A077939 in OEIS, [11].

TABLE 2. Some terms of the sequences $\left\{R_{n}\right\},\left\{S_{n}\right\},\left\{T_{n}\right\}$ and $\left\{U_{n}\right\}$

\begin{tabular}{||cccccccccccc||}
\hline $\mathrm{n}$ & 0 & 1 & 2 & 3 & 4 & 5 & 6 & 7 & 8 & 9 & 10 \\
\hline \hline$R_{n}$ & 3 & 2 & 6 & 17 & 42 & 107 & 273 & 695 & 1770 & 4508 & 11481 \\
\hline$S_{n}$ & 3 & 4 & 11 & 29 & 73 & 186 & 474 & 1207 & 3074 & 7829 & 19939 \\
\hline$T_{n}$ & 1 & 2 & 5 & 13 & 33 & 84 & 214 & 545 & 1388 & 3535 & 9003 \\
\hline$U_{n}$ & 0 & 2 & 5 & 12 & 31 & 79 & 201 & 512 & 1304 & 3321 & 8458 \\
\hline
\end{tabular}

By using the sequences $\left\{R_{n}\right\},\left\{S_{n}\right\}$ and $\left\{T_{n}\right\}$ applying induction over $n$, we obtain the followings:

Similarly, we get

$$
r_{1}^{n}=\frac{1}{3} R_{n}+\frac{1}{3} S_{n-1}(X+Y)+T_{n-2}\left(X^{2}+Y^{2}\right) .
$$

$$
r_{2}^{n}=\frac{1}{3} R_{n}-\frac{1}{6} S_{n-1}(X+Y)-\frac{1}{2} T_{n-2}\left(X^{2}+Y^{2}\right)+\sqrt{3} i\left[\frac{1}{6} S_{n-1}(X-Y)+\frac{1}{2} T_{n-2}\left(X^{2}+Y^{2}\right)\right],
$$

and

$$
r_{3}^{n}=\frac{1}{3} R_{n}-\frac{1}{6} S_{n-1}(X+Y)-\frac{1}{2} T_{n-2}\left(X^{2}+Y^{2}\right)-\sqrt{3} i\left[\frac{1}{6} S_{n-1}(X-Y)+\frac{1}{2} T_{n-2}\left(X^{2}+Y^{2}\right)\right],
$$

where $R_{n}$ denotes the $n^{\text {th }}$ term of $\left\{R_{n}\right\}, S_{n}$ denotes the $n^{\text {th }}$ term of $\left\{S_{n}\right\}$ and $T_{n}$ denotes the $n^{\text {th }}$ term of $\left\{T_{n}\right\}$. So, we calculate the powers of the roots $r_{1}^{n}, r_{2}^{n}$ and $r_{3}^{n}$ in terms of $R_{n}, S_{n}$ and $T_{n}$. Thus, we get the De Moivre-type identity for the third order Pell numbers.

\section{Generating Functions and Binet's Formula for the Sequences $\left\{R_{n}\right\},\left\{S_{n}\right\}$ and $\left\{T_{n}\right\}$}

The generating function for $\left\{R_{n}\right\}$ can be found as

$$
R(x)=\frac{3-4 x-x^{2}}{1-2 x-x^{2}-x^{3}}
$$

where $R(x)=\sum_{n=0}^{\infty} R_{n} x^{n}$, as in [12]. Similarly, the generating function for $\left\{S_{n}\right\}$ and $\left\{T_{n}\right\}$, can be calculated as follows;

and

$$
S(x)=\frac{3-6 x}{1-2 x-x^{2}-x^{3}},
$$

$$
T(x)=\frac{1}{1-2 x-x^{2}-x^{3}},
$$


where $S(x)=\sum_{n=0}^{\infty} S_{n} x^{n}$ and $T(x)=\sum_{n=0}^{\infty} T_{n} x^{n}$, respectively. Note that the expansion of the equation (4.1) was given as the the sequence A077939 in [11].

The Binet's formulas for $\left\{R_{n}\right\},\left\{S_{n}\right\}$ and $\left\{T_{n}\right\}$, by the help of the generating functions, can be found as follows:

$$
\begin{gathered}
R_{n}=r_{1}^{n}+r_{2}^{n}+r_{3}^{n}, \\
S_{n}=\frac{\left(3 r_{1}-6\right) r_{1}^{n+1}}{\left(r_{1}-r_{2}\right)\left(r_{1}-r_{3}\right)}+\frac{\left(3 r_{2}-6\right) r_{2}^{n+1}}{\left(r_{2}-r_{1}\right)\left(r_{2}-r_{3}\right)}+\frac{\left(3 r_{3}-6\right) r_{3}^{n+1}}{\left(r_{3}-r_{1}\right)\left(r_{3}-r_{2}\right)}, \\
T_{n}=\frac{r_{1}^{n+2}}{\left(r_{1}-r_{2}\right)\left(r_{1}-r_{3}\right)}+\frac{r_{2}^{n+2}}{\left(r_{2}-r_{1}\right)\left(r_{2}-r_{3}\right)}+\frac{r_{3}^{n+2}}{\left(r_{3}-r_{1}\right)\left(r_{3}-r_{2}\right)} .
\end{gathered}
$$

The equation (4) was obtained by Soykan [12].

\section{Some Properties of $\left\{R_{n}\right\},\left\{S_{n}\right\}$ ANd $\left\{T_{n}\right\}$}

In this section some interesting identities are derived as analogous to the idea in Ian Bruces' article [3], and by the help of the article [12], using the definition of the third order Pell numbers. The recurrence relations of the sequences $\left\{R_{n}\right\},\left\{S_{n}\right\},\left\{T_{n}\right\}$ and $\left\{U_{n}\right\}$ with initial conditions are follows:

$$
\begin{gathered}
R_{n+3}=2 R_{n+2}+R_{n+1}+R_{n}, n \geq 0, R_{0}=3, R_{1}=2, R_{2}=6, \\
S_{n+3}=2 S_{n+2}+S_{n+1}+S_{n}, n \geq 0, S_{0}=3, S_{1}=4, S_{2}=11, \\
T_{n+3}=2 T_{n+2}+T_{n+1}+T_{n}, n \geq 0, T_{0}=1, T_{1}=2, T_{2}=5 . \\
U_{n+3}=2 U_{n+2}+U_{n+1}+U_{n}, n \geq 0, U_{0}=0, U_{1}=2, U_{2}=5 .
\end{gathered}
$$

By using these equations, the following identities are hold:

$$
\begin{gathered}
U_{n}=2 T_{n-1}+T_{n-2}, n \geq 2 . \\
S_{n}=3 T_{n}-2 T_{n-1}, n \geq 1 . \\
R_{n}=2 T_{n-1}+2 T_{n-2}+3 T_{n-3}, n \geq 3 . \\
\sum_{k=0}^{n} U_{k}=T_{n+1}-T_{n}-1 . \\
\sum_{k=0}^{n} R_{k}=\frac{7 T_{n}-T_{n-1}-2 T_{n-2}+2}{3} . \\
\sum_{k=0}^{n} T_{k}=\frac{10 T_{n}+2 T_{n-1}+T_{n-2}+2}{3} .2 T_{n-1}+T_{n-2}-1 \\
3 \\
U_{n+1}^{2}+U_{n-1}^{2}=4 T_{n}^{2}+2 T_{n-1}^{2}+T_{n-4}^{2}+4 T_{n} T_{n-1}-2 T_{n-1} T_{n-4}, n \geq 5 . \\
U_{n+1} U_{n-1}=T_{n-1}^{2}+2 T_{n}\left(T_{n-1} T_{n-4}\right)-T_{n-1} T_{n-4}, n \geq 5 . \\
\sum_{n-1} .
\end{gathered}
$$




\section{Conclusion}

In this study, we give a way for constructing the Pell and Pell-Lucas numbers. Also, we obtain the De Moivretype identities, occupy an important place in the analysis, for the second and the third order Pell sequences. In future studies, this identity can be derived for the fourth order Pell numbers as similar to the De Moivre-type identity for the tetrabocacci numbers in the literature.

\section{ACKNOWLEDGEMENT}

The authors appreciate anonymous referees for their careful corrections to and valuable comments on the paper.

\section{CONFLICTS OF INTEREST}

The authors declare that there are no conflicts of interest regarding the publication of this article.

\section{REFERENCES}

[1] Aydın, F. G., Köklü, K., Yüce, S., Generalized dual Pell quaternions, Notes on Number Theory and Discrete Mathematics, 23(4)(2017), 66-84.

[2] Bicknell, M., Hoggatt, V. E., A primer for the Fibonacci numbers, Santa Clara, Calif.: The Fibonacci Association, B-10(1972), 45.

[3] Bruce, I., A modified Tribonacci sequence, The Fibonacci Quarterly 22, 3(1984), 244-246.

[4] Ercolano, J., Matrix generators of Pell sequences, The Fibonacci Quarterly, 17 (1)(1979), 71-77.

[5] Horadam, A. F., Pell identities, The Fibonacci Quarterly 26, 9(3)(1971), 245-252.

[6] Kılıç, E., Taşc1, D., The generalized Binet formula, representation and sums of the generalized order- Pell numbers, Taiwanese J. Math., 10 (6)(2006), 1661-1670.

[7] Kılıç, E., The generalized order- $k$ Fibonacci - Pell sequence by matrix methods, Journal of Computational and Applied Mathematics, 209(2)(2007), 133-145.

[8] Pethö, A., The Pell sequence contains only trivial perfect powers, In Colloquia on Sets, Graphs and Numbers, Soc. Math., János Bolyai, North-Holland, Amsterdam (1991), 561-568.

[9] Pin-Yen, L., De Moivre-type identities for the Tribonacci numbers, The Fibonacci Quarterly, 2(1988), 131-134.

[10] Pin-Yen, L., De Moivre-Type Identities for the Tetranacci Numbers, In: Bergum G.E., Philippou A.N., Horadam A.F. (eds) Applications of Fibonacci Numbers. Springer, Dordrecht(1991), https://doi.org/10.1007/978-94-011-3586-324.

[11] Sloane, N.J.A., The on-line encyclopedia of integer sequences, http://oeis.org/.

[12] Soykan, Y., On Generalized third-order Pell numbers, Asian Journal of Advanced Research and Reports, 6(1)(2019), https://doi.org/10.9734/ajarr/2019/v6i130144, 1-18. 